\title{
Robust Damping PI Repetitive Control for Nanopositioning
}

\author{
Arnfinn Aas Eielsen ${ }^{\dagger}$ and Jan Tommy Gravdahl ${ }^{\ddagger}$ \\ Department of Engineering Cybernetics \\ Norwegian University of Science and Technology \\ Trondheim, Norway
}

\author{
Kam K. Leang* \\ Mechanical Engineering Department \\ University of Nevada, Reno \\ Reno, NV 89557, USA
}

\begin{abstract}
In many applications of nanopositioning, such as scanning probe microscopy, tracking fast periodic reference trajectories with high accuracy is highly desirable. Repetitive control (RC) is a simple and effective scheme to obtain good tracking of such reference trajectories. However, the highly resonant dynamics of the positioning stage combined with hysteresis and creep behavior in the piezoelectric actuator can degrade performance and even make creating a stable RC system difficult. In this paper, a damping proportionalintegral (PI) controller is combined with a repetitive controller for robustness and high performance. Compared to a regular PI controller, the modified PI controller introduces damping, increases the bandwidth, and reduces the overall noise level due to feedback. Also, due to the integral action, the hysteresis and creep nonlinearities inherent in the piezoelectric actuator is minimized. A novel method for tuning the PI controller is proposed. The control approach is applied to a customdesign flexure-guided nanopositioning system with a dominant resonance of approximately $725 \mathrm{~Hz}$. Experimental results demonstrate the effectiveness of the overall control scheme, and the maximum tracking error for scanning at $100 \mathrm{~Hz}$ and $400 \mathrm{~Hz}$ is measured at $0.27 \%$ and $2.7 \%$, respectively, of the total positioning range.
\end{abstract}

\section{INTRODUCTION}

Nanopositioners employed in applications that include scanning probe microscopy require tracking of fast periodic reference trajectories with high accuracy [1], [2]. These positioning systems also involve moving payloads of various masses. The resonance frequencies and the effective control gain of the mechanical structure will therefore change every time a new payload is attached. Because the majority of nanopositioning designs use piezoelectric actuators, inherent variations in the effective control gain due to changes in actuator temperature, offset voltage, displacement range, as well as due to depolarization of the piezoelectric actuator must be taken into account. Piezoelectric actuators also introduce disturbances due to hysteresis and creep. Herein, a damping proportional-integral (PI) controller is combined with a repetitive controller (RC) and a novel tuning methodology is proposed. Compared to regular PI control, the proposed PI controller introduces damping, increases the bandwidth, and reduces the overall noise level due to feedback. The proposed PI controller utilizes the instrumentation already present in the signal chain, and provides a very low complexity control solution which is on par with more complex control schemes, such as controllers based on $H_{\infty}$ synthesis [3], although the latter can in principle provide higher bandwidth by attempting to control higher frequency dynamics. Additionally, the integral action helps to minimize the tracking error due to

\footnotetext{
†E-mail: eielsen@itk.ntnu.no.

‡E-mail: Jan. Tommy. Gravdahleitk. ntnu. no.

*Corresponding author; E-mail: kameunr.edu.
}

hysteresis and creep nonlinearities inherent in the piezoactuator. The proposed approach offers good asymptotic tracking and robustness for tracking periodic reference trajectories, and experimental results are presented to demonstrate the efficacy of the approach.

Recently, repetitive control has been introduced for nanopositioning systems [4]. The repetitive control scheme is based on the internal model principle [5] and it is specifically tailored to track periodic reference trajectories. At the heart of the controller is a signal generator that provides high gain at the harmonics of the reference trajectory. RC can be easily implemented digitally using a pure delay inside of a positive feedback loop [6]. Compared to traditional feedback and feedforward controllers [2], [7], the tracking error of RC diminishes as the number of operating periods increases. The controller generally requires only the period of the reference trajectory to be known [6]. In many nanopositioning applications (such as scanning probe microscopy), the period of the reference signal is known in advance which makes RC attractive. Compared to iterative learning control (ILC) [8][11], RC does not require resetting the initial conditions at the start of each iteration step. It is pointed out that ILC for hysteretic systems require a cycling process to reset the initial conditions at the beginning of each iteration [11]. For convenience, an RC can be plugged into an existing feedback loop to enhance performance.

The performance and stability of RC depends on the dynamics of the controlled system [12], [13]. Particularly, sharp resonance peaks can degrade performance and even make creating a stable RC system difficult. To provide more feasible system dynamics, a damping PI controller is proposed and combined with RC to minimize the effect of the highly resonant dynamics of the nanopositioning stage [14]. A novel tuning procedure is proposed to optimally tune the PI controller for robustness and good tracking performance. The control approach is applied to a custom-designed piezo-based nanopositioning system. Results are presented to demonstrate the effectiveness of the overall control scheme, where the maximum tracking error for scanning at $100 \mathrm{~Hz}$ and $400 \mathrm{~Hz}$ is measured at $0.27 \%$ and $2.7 \%$, respectively, of the total positioning range. The scheme also has very low computational requirements, where closed-loop sampling frequency of $100 \mathrm{kHz}$ can be obtained on a regular personal computer.

\section{SYSTEM DESCRIPTION AND MODELING}

The system under consideration consists of a customdesigned serial-kinematic nanopositioning stage, antialiasing and reconstruction filters, a piezo voltage amplifier, a capacitive displacement sensor, and a computer equipped with digital-to-analog and analog-to-digital converters for 


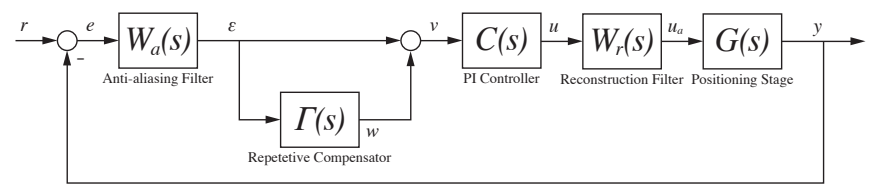

Fig. 1: System block diagram.

implementing the control scheme. The controller combines a plug-in repetitive controller and a PI feedback controller as shown in Fig. 1. As the amplifier and sensor used in the experimental set-up have very fast dynamics, they have been mostly neglected in the system analysis.

The nanopositioning stage used in this work is shown in Fig. 2, where the serial-kinematic motion mechanism is designed such that the first vibration mode is dominant and occurs in the actuation direction (piston mode). The simplified free body diagram for the mechanism is displayed in the inset image in Fig. 2, and by this model the corresponding second-order differential equation to describe the dynamics (where subscript " $x$ " indicates the $x$ direction) is given by

$$
m_{x} \ddot{x}_{x}(t)+c_{x} \dot{x}_{x}(t)+k_{x} x_{x}(t)=f_{x}(t),
$$

where $m_{x}(\mathrm{~kg})$ is the mass of the sample platform, as well as any additional mass due an attached payload, $c_{x}\left(\mathrm{~N} \mathrm{~s} \mathrm{~m}^{-1}\right)$ is the damping coefficient, and $k_{x}\left(\mathrm{~N} \mathrm{~m}^{-1}\right)$ is the spring constant. The applied external force from the piezoelectric actuator $f_{x}(\mathrm{~N})$ is

$$
f_{x}(t)=\beta u_{a}(t)+d_{h}(t),
$$

where $\beta\left(\mathrm{N} \mathrm{V}^{-1}\right)$ is the effective gain ${ }^{1}$ of the piezoelectric actuator from voltage to force, and $u_{a}(t)(\mathrm{V})$ is the applied voltage. The piezoelectric actuator will introduce hysteresis and creep when driven by a voltage signal. It is a reasonable assumption to consider this behavior as a bounded disturbance added to the input [15], represented by the term $d_{h}(t)$.

Denoting the output $y=x_{x}$, the transfer function for the nanopositioning stage is

$$
G(s)=\frac{y}{u_{a}}(s)=\frac{b_{0}}{s^{2}+a_{1} s+a_{0}}=\frac{b_{0}}{s^{2}+2 \zeta \omega_{0} s+\omega_{0}^{2}},
$$

where $b_{0}=\beta / m\left(\mathrm{~m} \mathrm{~s}^{-2} \mathrm{~V}^{-1}\right), a_{0}=k / m\left(\mathrm{~s}^{-2}\right), a_{1}=c / m$ $\left(\mathrm{s}^{-1}\right), \zeta=c / 2 \sqrt{m k}(-)$, and $\omega_{0}=\sqrt{k / m}\left(\mathrm{~s}^{-1}\right)$.

The frequency response for the $x$ axis is recorded using a SR785 Dynamic Signal Analyzer from Stanford Research Systems with bandwidth-limited white noise excitation. The response is displayed in Fig. 3. The model (3) is fitted to the frequency response data using the MATLAB System Identification Toolbox, and the resulting parameter values are: $b_{0}=2.81 \cdot 10^{6} \mu \mathrm{m} \mathrm{s}^{-2} \mathrm{~V}^{-1} ; a_{0}=2.08 \cdot 10^{7} \mathrm{~s}^{-2}$; $a_{1}=79.4 \mathrm{~s}^{-1} ; \zeta=8.72 \cdot 10^{-3} ;$ and $\omega_{0}=2 \pi \cdot 725 \mathrm{~s}^{-1}$. The response of the model (3) using these parameters is also displayed in Fig. 3 for comparison.

As can be seen, the actual response of the first vibration mode is well approximated by the second-order model (3). There are higher order modes in the system, and the second

\footnotetext{
${ }^{1}$ Mainly determined by the material, amount of polarization, and the driving voltage amplitude (as the amount of deflection generated changes with voltage amplitude due to hysteresis).
}

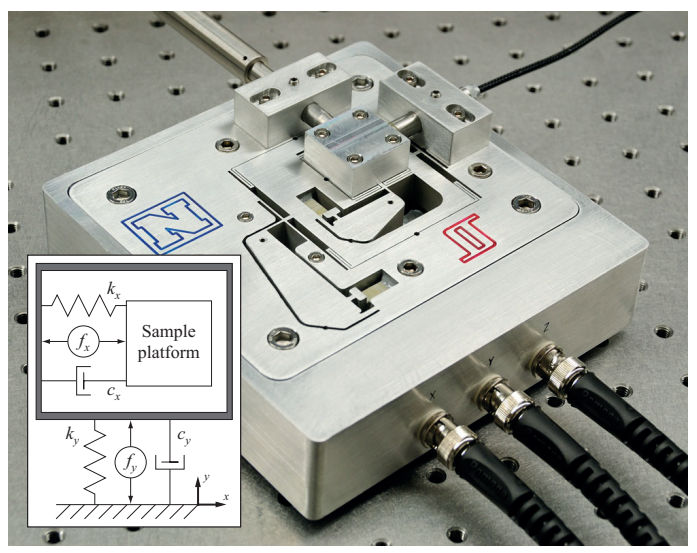

Fig. 2: Experimental serial-kinematic nanopositioning stage.

and third vibration modes are clearly visible in Fig. 3. The higher order modes have negligible magnitude responses in comparison to the first, thus the second-order model is sufficient to describe the dominant dynamics of the system. It should also be noted that higher order vibration modes are likely to have shapes and directions that will make them difficult to control using the mounted actuator.

As already discussed, the system has uncertainty with regards to the parameters, and the chosen model for the nanopositioning stage does not include high frequency dynamics. A reasonable stability margin is therefore needed to handle these uncertainties and the controller needs sufficient attenuation at higher frequencies to avoid excitation of the higher frequency dynamics of the nanopositioning stage. To assess the robustness of the proposed control scheme, the uncertainty of the system model is taken into account as a multiplicative perturbation to the positioner dynamics, i.e.,

$$
G_{p}(s)=G(s)\left(1+w_{G}(s) \Delta_{G}(s)\right) ;\left|\Delta_{G}(j \omega)\right| \leq 1 \forall \omega .
$$

The uncertainty weight $w_{G}(s)$ is determined experimentally, and an approximate overbound is found. These results are shown in Fig. 4.

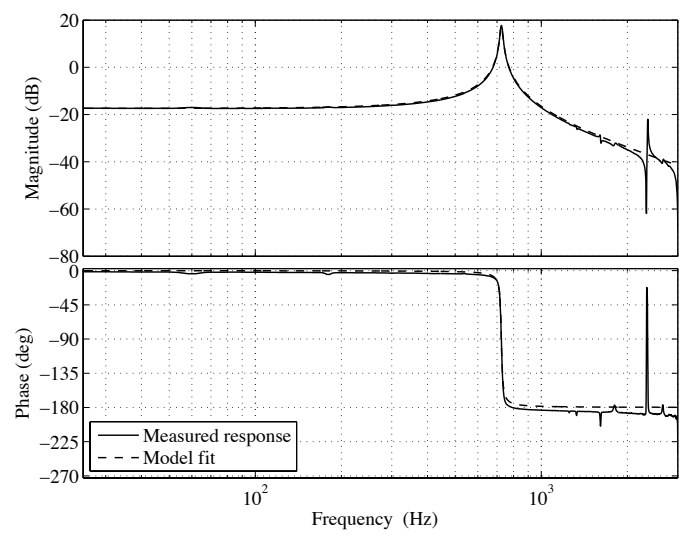

Fig. 3: Measured frequency response (solid line) for $x$ axis of the nanopositioning stage and the model (3) (dash line). 


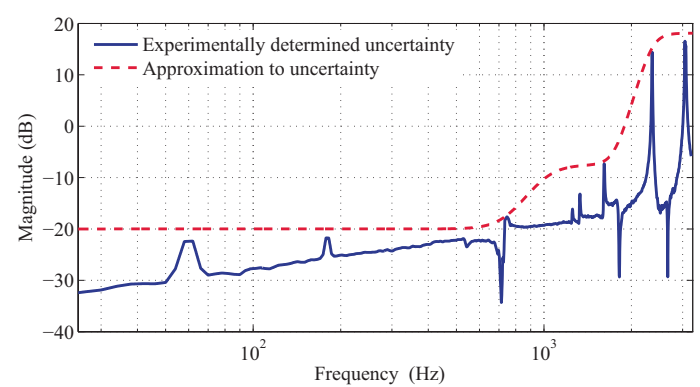

Fig. 4: Open-loop uncertainty weight $w_{G}(s)$, using experimental data and as overbounding transfer function.

\section{CONTROLLER DESIGN AND TUNING}

\section{A. Repetitive Controller}

Repetitive control intends to track or reject arbitrary periodic signals of a fixed period $T_{p}$, by embedding a model of the reference $r$ or disturbance $d$ signal in the controller. The transfer function for the RC configuration shown in Fig. 5(a) is given as

$$
\frac{w}{\varepsilon}(s)=\Gamma(s)=\frac{R(s) \mathrm{e}^{-\tilde{T}_{p} s}}{1-Q(s) \mathrm{e}^{-\tilde{T}_{p} s}},
$$

where $Q(s)$ is a unity-gain low-pass filter, and $R(s)$ is an output filter, defined below. It is pointed out that the low pass filter $Q(s)$ inherently shifts all the poles into the complex left half-plane, with an amount dependent on frequency, so at higher frequencies the new pole location is father away from the original location than at lower frequencies. This will degrade the nulling property of the controller at the fundamental and harmonic frequencies of the reference signal. A modified $\tilde{T}_{p}$ is introduced to obtain some compensation for the shift in pole locations due to $Q(s)$. A method for minimizing the tracking error by tuning $\tilde{T}_{p}$ is given in Section III-F.

By inspection of Fig. 1, the closed-loop sensitivity function for the overall system is found as

$$
\frac{e}{r}(s)=S(s)=\frac{1}{1+\bar{G}(s) C(s)+\bar{G}(s) C(s) \Gamma(s)}=\frac{1}{\Delta(s)},
$$

where $\bar{G}(s)=W_{r}(s) G(s) W_{a}(s)$. The stability of the closedloop system is determined by the denominator

$$
\Delta(s)=1+\bar{G}(s) C(s)+\bar{G}(s) C(s) \Gamma(s) .
$$

Now, consider the sensitivity $\bar{S}(s)$ and complementary $\bar{T}(s)$ sensitivity function without the presence of the repeti-

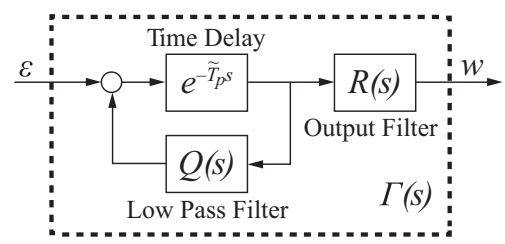

(a)

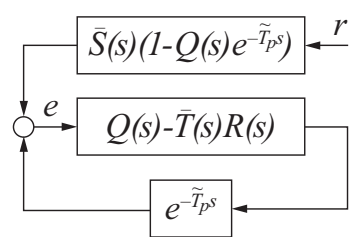

(b)
Fig. 5: (a) Plug-in repetitive controller. (b) Equivalent representation of the sensitivity function Eq. (9). tive controller

$$
\bar{S}(s)=\frac{1}{1+C(s) \bar{G}(s)} \text { and } \bar{T}(s)=C(s) \bar{G}(s) \bar{S}(s) .
$$

Then, by inserting the expression for $\Gamma(s)$, multiplying the numerator and denominator of $1 / \Delta(s)$ by $\bar{S}(s)$, and rearranging, the sensitivity function for the closed-loop system when adding the repetitive controller becomes

$$
S(s)=\frac{1}{\Delta(s)}=\frac{\bar{S}(s)\left(1-Q(s) \mathrm{e}^{-\tilde{T}_{p} s}\right)}{1-(Q(s)-\bar{T}(s) R(s)) \mathrm{e}^{-\tilde{T}_{p} s}} .
$$

With reference to Fig. 5(b), it can be seen that given a bounded reference $r(t)$ and stable transfer functions $\bar{S}(s)$ and $Q(s)$, the small-gain theorem provides the criterion for the stability of the closed-loop system as [16]

$$
\|Q(s)-\bar{T}(s) R(s)\|_{\infty}<1
$$

where it is noted that $\left|\mathrm{e}^{-j \tilde{T}_{p} \omega}\right|=1 \forall \omega \in \mathbb{R}$.

The output filter $R(s)$ is constructed as

$$
R(s)=W_{T}^{-1}(s) Q(s),
$$

introducing a stable all-pole filter $W_{T}(s)$, which provides for some flexibility in meeting the stability criterion. Using a unity-gain low-pass filter $Q(s)$, the following somewhat simpler criterion

$$
\left\|1-W_{T}^{-1}(s) \bar{T}(s)\right\|_{\infty}<1
$$

can be used for selecting an appropriate filter $W_{T}(s)$.

To assess the robustness of the $\mathrm{RC}$, a multiplicative perturbation for the closed loop complementary sensitivity is used, that is:

$$
\bar{T}_{p}(s)=\bar{T}(s)\left(1+w_{T}(s) \Delta_{T}(s)\right) ;\left|\Delta_{T}(j \omega)\right| \leq 1 \forall \omega,
$$

where

$$
\begin{aligned}
w_{T}(s) \Delta_{T}(s)= & \frac{\bar{S}(s) w_{G}(s) \Delta_{G}(s)}{1+\bar{T}(s) w_{G}(s) \Delta_{G}(s)} \Rightarrow \\
& \left|w_{T}(j \omega)\right| \leq\left|\bar{S}(j \omega) w_{G}(j \omega)\right| .
\end{aligned}
$$

Incorporating the uncertainty into the criterion (10), we obtain the robust stability criterion:

$$
|Q(j \omega)-\bar{T}(j \omega) R(j \omega)|<1-\left|\bar{T}(j \omega) w_{T}(j \omega) R(j \omega)\right| .
$$

\section{B. Damping Proportional Integral Controller}

The nanopositioning stage is a lightly damped structure as shown by the measured frequency response in Fig. 3. Inspecting the stability criterion for the repetitive controller, one can expect that large peaks in the complementary sensitivity function $\bar{T}(s)$ can reduce the applicable bandwidth and gain for the repetitive controller, depending on how well the output filter $R(s)$ is able to match the inverse closedloop dynamics. Introducing a robust damping and tracking controller should increase the robustness and bandwidth possible for the overall control scheme.

A simple, effective, and robust damping and tracking controller for a lightly damped structure can be obtained by modifying and optimally tuning a proportional integral (PI) controller. Since the controller is implemented in discrete 
time using digital-to-analog and analog-to-digital converters, reconstruction and anti-aliasing filters must be present in order to mitigate anti-aliasing effects and quantization noise. By including these filters in the controller, an extra degree of freedom can be added for the tuning of the controller, which is the cut-off frequency of the filters. Compared to a regular PI controller the modified PI controller will introduce damping, increase the bandwidth, and reduce the overall noise level due to feedback. Also, due to the integral action, the hysteresis and creep nonlinearities introduced by the piezoelectric actuator will be effectively suppressed.

A PI controller is typically given as

$$
C(s)=\frac{k_{p} s+k_{i}}{s},
$$

where $k_{p}$ and $k_{i}$ are the proportional and integral gain, respectively. Here the filters are taken to be second-order low-pass Butterworth filters

$$
W_{a}(s)=W_{r}(s)=\frac{\omega_{c}^{2}}{s^{2}+\sqrt{2} \omega_{c} s+\omega_{c}^{2}},
$$

where $\omega_{c}$ is the cut-off frequency. For convenience they are taken to be identical when tuning the controller. The modified PI controller is therefore

$$
\bar{C}(s)=W_{a}(s) C(s) W_{r}(s) .
$$

\section{Proportional Integral Controller Tuning}

The procedure for obtaining the optimal tuning of the PI controller follows the work in [14]. First, consider an integral controller

$$
C_{I}(s)=k_{i} \frac{1}{s}
$$

applied to (3). The stability margin $k_{i}^{*}$ using the RouthHurwitz criterion can be found to be

$$
k_{i}<\frac{a_{0} a_{1}}{b_{0}}=\frac{2 \zeta \omega_{0}^{3}}{\beta_{0}}=k_{i}^{*} .
$$

Given a lightly damped system, $0<\zeta \ll 1 / \sqrt{2}$, knowing the resonance frequency $\omega_{p}$ and the amplitude response at resonance $\left|G\left(j \omega_{p}\right)\right|$,

$$
k_{i}<\frac{\omega_{p}}{\left|G\left(j \omega_{p}\right)\right|}<\kappa \cdot \frac{\omega_{p}}{\left|G\left(j \omega_{p}\right)\right|}=k_{i}^{*},
$$

where $\kappa^{-1}=\sqrt{1-\zeta^{2}} \cdot \sqrt{1-2 \zeta^{2}}<1$.

By adding proportional action to the above controller, the effective stiffness of the system increases. The product $\zeta \omega_{0}{ }^{3}$ will therefore be larger, and thus the margin becomes larger.

Considering (20), it can be conjectured that in order to increase the bandwidth of the controller, introducing damping, i.e. reducing $\left|G\left(j \omega_{p}\right)\right|$, would be effective.

\section{Effects of Low-Pass Filters in the Signal Chain}

The modified PI controller (18) has an extra degree of freedom, that is in the choice of the cut-off frequency of the filters (17). By adjusting the cut-off frequency $\omega_{c}$, damping can be introduced and the overall bandwidth can be increased. This is illustrated in Fig. 6. Setting the control gains to $k_{p}=1$ and $k_{i}=k_{i}^{*}=588$, i.e., setting the PI-controller gains close to the margin of stability, the eigenvalue loci of

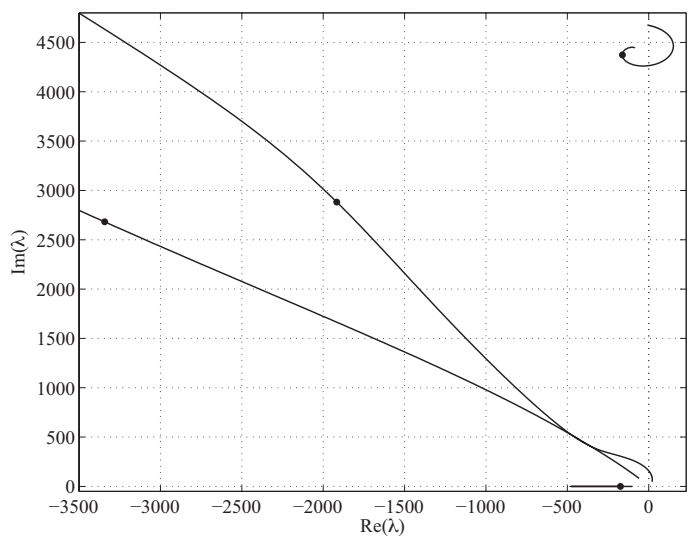

Fig. 6: Eigenvalue loci for the closed-loop system as a function of the filter cut-off frequency $\omega_{c}$. The loci are symmetric about the real axis. The optimal root locations according to (21) are indicated by dots.

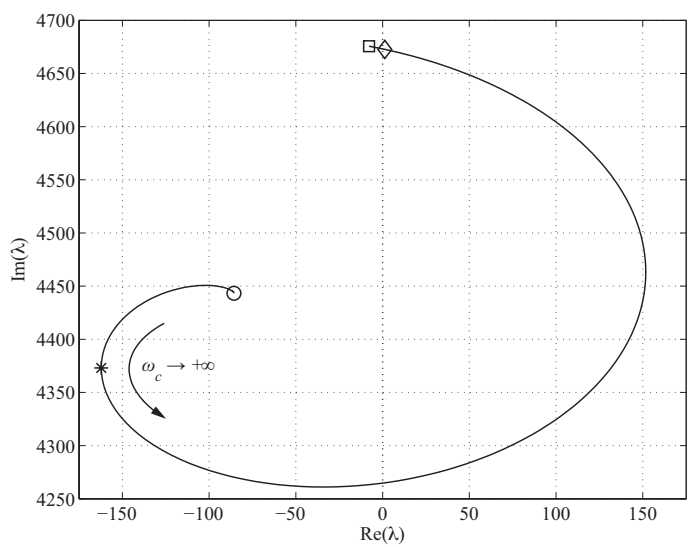

Fig. 7: Detail from the upper-right corner of Fig. 6. The eigenvalue when $\omega_{c} \rightarrow 0$ is indicated by a circle, $\omega_{c}=\omega_{c}^{\dagger}$ a star, $\omega_{c}=\omega_{n}$ a diamond shape, and $\omega_{c} \rightarrow+\infty$ a square.

the closed-loop system is generated by varying the cut-off frequency $\omega_{c}$ of the reconstruction and anti-aliasing filters.

As can be seen from the detail in Fig. 7, using filters with a cut-off frequency equal to the Nyquist frequency, $\omega_{c}=$ $\omega_{n}=2 \pi f_{n}$, where $f_{n}=50 \mathrm{kHz}$, the system is unstable. The Figs. 6 and 7 indicate, however, that reducing the cutoff frequency for the filters, all eigenvalues can be moved further into the left-half plane. Minimizing the maximum value of the real part of the eigenvalues for the closed-loop system as a function of the cut-off frequency $\omega_{c}$ resulted in

$$
\begin{aligned}
& \omega_{c}^{\dagger}=\underset{\omega_{c} \in \mathbb{R}^{+} /\{0\}}{\arg \min }\left[\arg \max \left[\operatorname{det}\left(A\left(\omega_{c}\right)-\lambda I\right)=0\right]\right] \approx \\
& \omega_{c} \in \mathbb{R}^{+} /\{0\} \operatorname{Re}(\lambda) \in \mathbb{R}^{-} \\
& 2 \pi \cdot 610 \mathrm{rad} \mathrm{s}^{-1},
\end{aligned}
$$

where $A\left(\omega_{c}\right)$ is a state matrix realization for the closed-loop system for a given $\omega_{c}$. Thus, setting $\omega_{c}=\omega_{c}^{\dagger}=2 \pi f_{o}$, where $f_{o}=610 \mathrm{~Hz}$, will produce the fastest modes for this system with the selected control gains.

By inspecting Figs. 6 and 7, it is evident that by using the computed optimal value for the cut-off frequency, the stability margins for the closed-loop system will be larger. This 


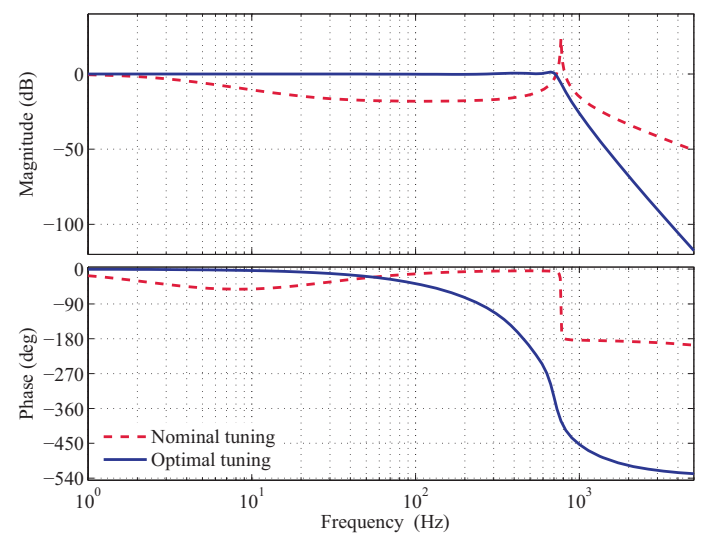

Fig. 8: Amplitude and phase response for the complementary sensitivity function $\bar{T}(s)=\frac{y}{r}(s)$ (PI control), using nominal and optimal tuning.

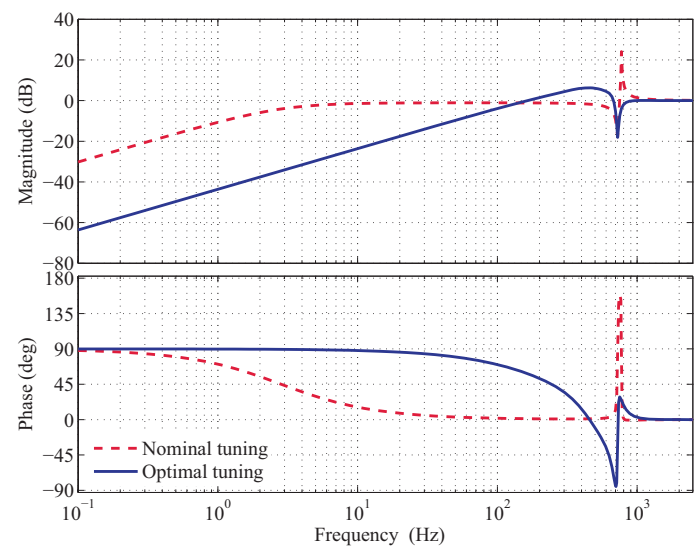

Fig. 9: Amplitude and phase response for the sensitivity function $\bar{S}(s)=\frac{e}{r}(s)$ (PI control), using nominal and optimal tuning.

should allow for a larger closed-loop bandwidth. The new stability margin is found numerically to be about $23.9 \cdot k_{i}^{*}$. As the optimal cut-off frequency is somewhat sensitive to the choice of control gains, the optimal values for both the cut-off frequency and the integral gain is found minimizing

$$
J_{C}\left(k_{i}, \omega_{c}\right)=\left\|1-\left|\bar{T}\left(j \omega, k_{i}, \omega_{c}\right)\right|\right\|_{2},
$$

attempting to produce the flattest possible response for the complementary sensitivity function. As larger values of $k_{p}$ resulted poorer closed-loop response, this gain value is set to a fixed low value of $k_{p}=1 \mathrm{~V} / \mu \mathrm{m}$.

Evaluating the cost-function (22) resulted in

$$
\begin{aligned}
{\left[k_{i}^{\dagger}, \omega_{c}^{\dagger}\right]=} & \underset{k_{i} \in\left[0,23.9 \cdot k_{i}^{*}\right], \omega_{c} \in \mathbb{R}^{+} /\{0\}}{\arg \min }\left[J_{C}\left(k_{i}, \omega_{c}\right)\right] \approx \\
& {\left[7050 \mathrm{~V} \mathrm{~s}^{-1} \mu \mathrm{m}^{-1}, 2 \pi \cdot 728 \mathrm{rad} \mathrm{s}^{-1}\right] . }
\end{aligned}
$$

Using the optimal cut-off frequency and integral gain should produce the flattest frequency response when using a PIcontroller, and it yielded a gain margin of $\Delta K=5.78 \mathrm{~dB}$ and a phase margin of $\Psi=61.6^{\circ}$.

This result can be compared to a nominal tuning, which corresponds to reconstruction and anti-alias filters using $\omega_{c}=\omega_{n}$, and tuning the PI controller gains to match the gain margin of the optimal tuning. Using $k_{p}=1$ and $k_{i}=150$ yields $\Delta K=5.71 \mathrm{~dB}$ and $\Psi=97.7^{\circ}$. The resulting frequency responses for the complementary sensitivity and sensitivity for the nominal and optimal configurations are displayed in Fig. 8 and 9. The optimal tuning effectively attenuates the peak in Fig. 8, from $24 \mathrm{~dB}$ in the nominal case, to $1.3 \mathrm{~dB}$ in the optimal case, and by inspection of Fig. 9 a significant increase in closed-loop bandwidth can be seen; from approximately $4 \mathrm{~Hz}$ in the nominal case to approximately $100 \mathrm{~Hz}$ in the optimal case. The increase in bandwidth provides better tracking performance and suppression of input disturbances, such as due to the hysteresis effect.

The robustness of the PI with regards to the uncertainties displayed in Fig. 4 is determined. The criterion [17]

$$
\left\|w_{G}(s) \bar{T}(s)\right\|_{\infty}<1=0 \mathrm{~dB}
$$

is evaluated, and the results are shown in Fig. 10.

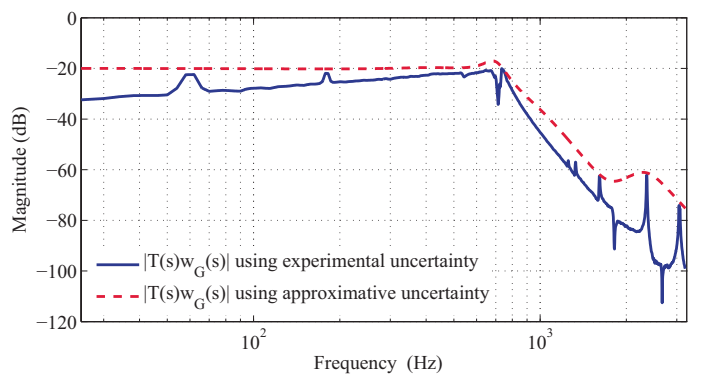

Fig. 10: Evaluation of robust stability criterion $\left|w_{G}(j \omega) \bar{T}(j \omega)\right|$ for the modified PI controller.

\section{E. Choosing $R(s)$ and $Q(s)$}

Considering the stability criterion for the repetitive controller (12), the ideal choice for the included filter would be $W_{T}(s)=\bar{T}(s)$, as this would produce the minimum of the norm (12). A sufficient choice for $W_{T}(s)$ would be a filter that attenuates $\bar{T}(s)$ sufficiently to meet the stability criterion.

The complementary sensitivity function $\bar{T}(s)$ has relative degree $n_{r}=6$. As the closed loop response of the system using the modified PI controller is fairly well behaved, $W_{T}(s)$ is chosen to be a sixth-order Butterworth filter with dc-gain $k_{T}$, and it is assumed that $W_{T}(s) \approx \bar{T}(s)$. An optimal choice for the cut-off frequency $\omega_{T}$ and dc-gain $k_{T}$ for $W_{T}(s)$ is found minimizing the cost-function

$$
J_{T}\left(k_{T}, \omega_{T}\right)=\left\|1-W_{T}^{-1}\left(s, \omega_{T}, k_{T}\right) \bar{T}(s)\right\|_{\infty},
$$

which resulted in $k_{T}^{\dagger} \approx 0.43$ and $\omega_{T}^{\dagger} \approx 2 \pi \cdot 570$.

The filter $Q(s)$ is now chosen, in consideration of the filter $R(s)$, to be unity-gain sixth-order Butterworth filter, such that $R(s)=W_{T}{ }^{-1} Q(s)$ would be a realizable filter.

In order to obtain robust stability, the criterion in (15) is evaluated in order to find a suitable cut-off frequency for $Q(s)$, in order to attenuate the second and higher order vibration modes. A cut-off frequency $\omega_{Q}=2 \pi \cdot 2000 \mathrm{rad} \mathrm{s}^{-1}$ provided sufficient attenuation. Evaluation of (15) also suggested that $k_{T}$ and $\omega_{T}$ should be reduced somewhat to 
$k_{T}=0.3$ and $\omega_{T}=2 \pi \cdot 550$. The closed-loop uncertainties used are shown in Fig. 11, and the evaluation of (15) is shown in Fig. 12.

\section{F. Tuning of $\tilde{T}_{p}$}

Due to the low-pass filter $Q(s)$, perfect tracking is not possible, since

$$
\mathcal{L}^{-1}\left[\left(1-Q(s) \mathrm{e}^{-\tilde{T}_{p} s}\right) r(s)\right]=r(t)-\tilde{r}\left(t-T_{p}\right)
$$

where $\tilde{r}(t)=Q(t) * r(t) \neq r(t)$. As noted previously, by modifying the time-delay $\bar{T}_{p}$, the shift in the poles due to $Q(s)$ can be partially compensated for.

By filtering the intended reference signal by

$$
\tilde{Z}(s)=1-Q(s) \mathrm{e}^{-\tilde{T}_{p} s},
$$

the amount of suppression of frequency components of the reference signal can be numerically evaluated. By adjusting $\tilde{T}_{p}=T_{p}-\delta$ by varying $\delta$, an optimal $\delta$ that produces the best tracking performance can be found, that is, minimizing

$$
J(\delta)=\left\|\mathcal{L}^{-1}[\tilde{Z}(s, \delta)] * r(t)\right\|_{2}, \quad t \in\left[T_{p} m, T_{p}(m+1)\right]
$$

where $\delta \in\left(0, T_{p}\right)$, and $m>0$ is an integer number used to make $T_{p} m$ large enough for the transient response of $Q(s)$ to have died out. Using, e.g., $T_{p}=1 / f_{p}=1 / 50 \mathrm{~s}$, $\delta^{\dagger}=3.075639 \cdot 10^{-4} \mathrm{~s}$. Results for other $T_{p}$ are presented in Table I.

TABLE I: Table of optimal $\delta$ at various reference frequencies using $Q(s)$ with $\omega_{Q}=2 \pi \cdot 2000 \mathrm{rad} / \mathrm{s}$.

\begin{tabular}{|c|c|c|c|}
\hline$f_{p}$ & $\delta^{\dagger}$ & $f_{p}$ & $\delta^{\dagger}$ \\
\hline $20 \mathrm{~Hz}$ & $3.074803 \cdot 10^{-4} \mathrm{~s}$ & $100 \mathrm{~Hz}$ & $3.078570 \cdot 10^{-4} \mathrm{~s}$ \\
\hline $25 \mathrm{~Hz}$ & $3.074888 \cdot 10^{-4} \mathrm{~s}$ & $200 \mathrm{~Hz}$ & $3.090099 \cdot 10^{-4} \mathrm{~s}$ \\
\hline $50 \mathrm{~Hz}$ & $3.075639 \cdot 10^{-4} \mathrm{~s}$ & $400 \mathrm{~Hz}$ & $3.113085 \cdot 10^{-4} \mathrm{~s}$ \\
\hline
\end{tabular}

\section{EXPERIMENTAL RESULTS}

\section{A. Description of the Experimental System}

The experimental set-up consisted of a Dell Optiplex GX280 computer equipped with a PCI-6221 data acquisition board from National Instruments, running the XPC Target real-time operating system for hardware-in-the-loop simulations, a ADE 5130/5300 capacitive gauge and ADE 5501 capacitive probe from ADE Technologies, a PAD129

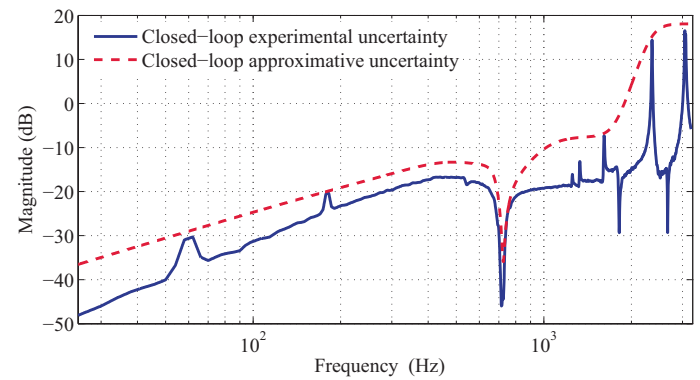

Fig. 11: Closed-loop uncertainty weight $w_{T}(s)$, using experimental data and as an overbounding transfer function. high-power operational (voltage) amplifier from PowerAmp Design, a custom-made long-range serial-kinematic nanopositioner, as well as two second-order low-pass Butterworth filters implemented using Twin-T topology, with Burr-Brown OPA2111 operational amplifiers and 10\% tolerance components, which are used as anti-aliasing and reconstruction filters.

Due to the $10 \%$ tolerance components used in the antialiasing and reconstruction filters, the frequency response of each of these filters are recorded and fitted to a second-order model, in order to obtain the best possible accuracy when tuning the controllers, and generating the reference signal.

With the xPC Target, a minimum sampling time of $T_{s}=$ $1 \cdot 10^{-5}\left(f_{s}=100 \mathrm{kHz}\right)$ is achieved and used for all the experiments.

\section{B. Results}

The results from the experiments are presented in Fig. 13 for scanning at $200 \mathrm{~Hz}$. The plots show the initial transient response of the measured deflection $y_{m}(s)=W_{a}(s) y(s)$ and error $\varepsilon(s)=W_{a}(s) r(s)-y_{m}(s)$, the steady-state measured deflection and error, as well as the steady-state control input $u$ and a power spectral density estimate of the steady-state error. A summary of all the experiments performed are presented in Table II. The experiments are mostly identical except for the fundamental frequency of the reference signal, a modified triangle wave. For all the experiments $10 \%$ of the reference signal is replaced with a interpolating polynomial to make it sufficiently smooth.

\section{DISCUSSION}

The results of the experiments in Table II are rated using both the maximum error (ME) and the root-mean-square error (RMSE), both in absolute terms with respect to the measured deflection, and relative to the maximum value of the reference signal. The quiescent noise in the system is measured to obtain an estimate of the noise floor of the system. This would suggest that the smallest achievable absolute ME would be $0.0168 \mu \mathrm{m}$, and the smallest absolute RMSE would be $0.00155 \mu \mathrm{m}$.

As can be seen from Table II, the closed loop error using references with low fundamental frequency yields errors close to the measurement noise. Some of the error is likely due to quantization noise. The ME appears to be lower than the measured noise floor, but this could possibly be because of changes in the noise environment.

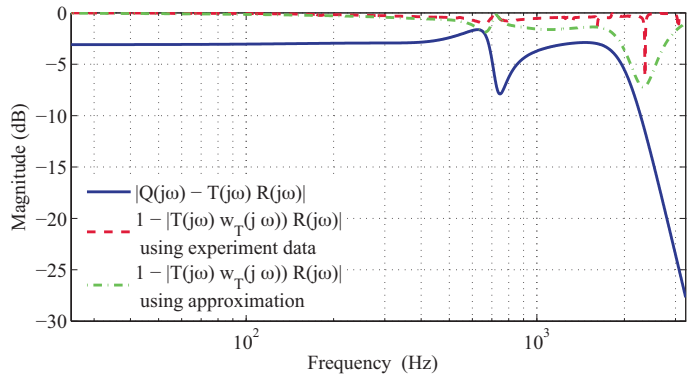

Fig. 12: Numeric evaluation of stability criteria (10) and (15). 
TABLE II: Norms of the measured error $\varepsilon$ in steady-state, using various configurations and reference signals. $f_{p}$ and $\alpha$ denotes the fundamental frequency and amplitude, respectively, of the reference signal.

\begin{tabular}{|c|c|c|c|c|c|}
\hline$f_{p}$ & $\alpha$ & $\begin{array}{c}\text { ME } \\
\text { absolute }\end{array}$ & $\begin{array}{c}\text { ME } \\
\text { relative }\end{array}$ & $\begin{array}{c}\text { RMSE } \\
\text { absolute }\end{array}$ & $\begin{array}{c}\text { RMSE } \\
\text { relative }\end{array}$ \\
\hline \hline \multicolumn{6}{|c|}{ When using PI controller and repetitive controller. } \\
\hline $20 \mathrm{~Hz}$ & $14.25 \mu \mathrm{m}$ & $0.013 \mu \mathrm{m}$ & $0.092 \%$ & $0.002 \mu \mathrm{m}$ & $0.024 \%$ \\
\hline $25 \mathrm{~Hz}$ & $14.25 \mu \mathrm{m}$ & $0.012 \mu \mathrm{m}$ & $0.085 \%$ & $0.0023 \mu \mathrm{m}$ & $0.027 \%$ \\
\hline $50 \mathrm{~Hz}$ & $14.25 \mu \mathrm{m}$ & $0.016 \mu \mathrm{m}$ & $0.11 \%$ & $0.0041 \mu \mathrm{m}$ & $0.048 \%$ \\
\hline $100 \mathrm{~Hz}$ & $14.25 \mu \mathrm{m}$ & $0.037 \mu \mathrm{m}$ & $0.27 \%$ & $0.013 \mu \mathrm{m}$ & $0.15 \%$ \\
\hline $200 \mathrm{~Hz}$ & $14.25 \mu \mathrm{m}$ & $0.12 \mu \mathrm{m}$ & $0.87 \%$ & $0.053 \mu \mathrm{m}$ & $0.62 \%$ \\
\hline $200 \mathrm{~Hz}$ & $1.425 \mu \mathrm{m}$ & $0.013 \mu \mathrm{m}$ & $0.95 \%$ & $0.0056 \mu \mathrm{m}$ & $0.66 \%$ \\
\hline $400 \mathrm{~Hz}$ & $7.600 \mu \mathrm{m}$ & $0.17 \mu \mathrm{m}$ & $2.7 \%$ & $0.11 \mu \mathrm{m}$ & $2.5 \%$ \\
\hline \hline \multicolumn{7}{|c|}{ When using PI controller. } \\
\hline $20 \mathrm{~Hz}$ & $14.25 \mu \mathrm{m}$ & $1.3 \mu \mathrm{m}$ & $8.9 \%$ & $0.83 \mu \mathrm{m}$ & $9.6 \%$ \\
\hline $200 \mathrm{~Hz}$ & $14.25 \mu \mathrm{m}$ & $15 \mu \mathrm{m}$ & $110 \%$ & $9.7 \mu \mathrm{m}$ & $110 \%$ \\
\hline
\end{tabular}

As the fundamental frequency increases, the error becomes large, as would be expected, since the bandwidth of the RC is mostly determined by the filter $Q(s)$. This is demonstrated by the PSD estimate for the error signal in Fig. 13c, which has very prominent peaks at odd harmonics of the fundamental frequency up to the bandwidth of $Q(s)$. Inspecting Fig. 13a, the transient response and convergence to steady state is clearly visible. The initial error is large because the reference signal fundamental frequency is above the bandwidth of the PI controller, and the RC controller requires about 5 cycles to converge. From Fig. 13d it can be seen that the the maximum error occurs at the maxima and minima of the reference signal, thus, increasing the linear proportion of the reference signal will also increase the maximum error, which is to be expected. The voltage range of the signal in Fig. 13e should also be noted, as most of the full range of the amplifier, $\pm 100 \mathrm{~V}$, is used, and it provides a hard limit with regards to the spectral content (i.e., the linear proportion) and the range of the reference signal, as well as the bandwidth of the controller. Increasing either one of these properties in this case, would have resulted in saturation in the amplifier.

\section{CONCLUSIONS}

It has been demonstrated through experiments that by using a damping PI controller and a well configured plugin repetitive control scheme, it is possible to achieve a maximum error of less than $1 \%$ relative to the reference signal, at reference signal frequencies exceeding $25 \%$ of the first resonance frequency of a nanopositioning stage. The methods used are straight-forward to implement and tune. Additionally, the control scheme is computationally light, and is therefore well suited for implementation on both micro-controllers and field-programmable-arrays. The proposed scheme can also be implementable on existing hardware configurations for nanopositioning, as it requires very small, or no, modifications to a standard configuration.

\section{ACKNOWLEDGMENTS}

This work was supported by the Norwegian University of Science and Technology, and in part by the National Science Foundation grant CMMI No. 0910570. Experiments were performed at the Electroactive Systems and Controls Lab (easyLab) at the University of Nevada, Reno. Authors thank Brian J. Kenton for his help with developing the flexurebased nanopositioning stage used in the experiments.

\section{REFERENCES}

[1] S. M. Salapaka and M. V. Salapaka, "Scanning probe microscopy," IEEE Control Systems Magazine, vol. 28, no. 2, pp. 65 - 83, 2008.

[2] G. M. Clayton, S. Tien, K. K. Leang, Q. Zou, and S. Devasia, "A review of feedforward control approaches in nanopositioning for high speed SPM,” ASME J. Dyn. Syst. Meas. and Cont., Special issue on Dynamic Modeling, Control, and Manipulation at the Nanoscale, vol. 131, no. 6, p. 061101 (19 pages), 2009.

[3] C. Lee and S. M. Salapaka, "Robust broadband nanopositioning: fundamental trade-offs, analysis, and design in a two-degree-of-freedom control framework," Nanotechnology, vol. 20, no. 3, p. 035501 (16 pages), 2008.

[4] U. Aridogan, Y. Shan, and K. K. Leang, "Design and analysis of discrete-time repetitive control for scanning probe microscopes," ASME J. Dyn. Syst. Meas. and Cont., Special issue on Dynamic Modeling, Control, and Manipulation at the Nanoscale, vol. 131, p. 061103 (12 pages), 2009.

[5] B. A. Francis and W. M. Wonham, "The internal model principle of control theory," Automatica, vol. 12, no. 5, pp. 457 - 465, 1976.

[6] T. Inoue, M. Nakano, and S. Iwai, "High accuracy control of a proton synchrotron magnet power supply," in Proc. 8th World Congr. IFAC, vol. 20 , pp. $216-221$.

[7] S. Devasia, E. Eleftheriou, and S. O. R. Moheimani, "A survey of control issues in nanopositioning," IEEE Trans. Cont. Syst. Tech., vol. 15 , no. 5 , pp. $802-823,2007$.

[8] K. L. Moore, M. Dahleh, and S. P. Bhattacharyya, "Iterative learning control: a survey and new results," Journal of Robotic Systems, vol. 9, no. 5, pp. 563-594, 1992.

[9] D. A. Bristow, J. Dong, A. G. Alleyne, P. Ferriera, and S. Salapaka, "High bandwidth control of precision motion instrumentation," Rev. Sci. Instr., vol. 79, p. 103704, 2008.

[10] Y. Wu and Q. Zou, "Iterative control approach to compensate for both the hysteresis and the dynamics effects of piezo actuators," IEEE Control Systems Technology, vol. 15, no. 5, pp. 936 - 944, 2007.

[11] K. K. Leang and S. Devasia, "Design of hysteresis-compensating iterative learning control for piezo positioners: application to atomic force microscopes," Mechatronics, vol. 16, no. 3-4, pp. $141-158$, 2006.

[12] S. Hara, Y. Yamamoto, T. Omata, and M. Nakano, "Repetitive control system: a new type servo system for periodic exogenous signals," IEEE Trans. Autom. Cont., vol. 33, no. 7, pp. 659 - 668, 1988.

[13] T. Inoue, "Practical repetitive control system design," in Proceedings of the 29th IEEE Conference on Decision and Control, vol. 3, pp. $1673-1678$.

[14] A. A. Eielsen, M. Burger, J. T. Gravdahl, and K. Y. Pettersen, " $\mathrm{PI}^{2}$ Controller Applied to a Piezoelectric Nanopositioner Using Conditional Integrators and Optimal Tuning," in 18th IFAC World Congress, Proceedings of the, 2011.

[15] A. A. Eielsen, J. T. Gravdahl, K. Y. Pettersen, and L. Vogl, "Tracking Control for a Piezoelectric Nanopositioner using Estimated States and Feedforward Compensation of Hysteresis," in 5th IFAC Symposium on Mechatronic Systems, Proceedings of the, 2010.

[16] S. Hara, Y. Yamamoto, T. Omata, and M. Nakano, "Repetitive control system: a new type servo system for periodic exogenous signals," Automatic Control, IEEE Transactions on, vol. 33, no. 7, pp. 659668, 1988.

[17] S. Skogestad and I. Postlethwaite, Multivariable Feedback Control: Analysis and Design. Wiley-Interscience, 2005. 

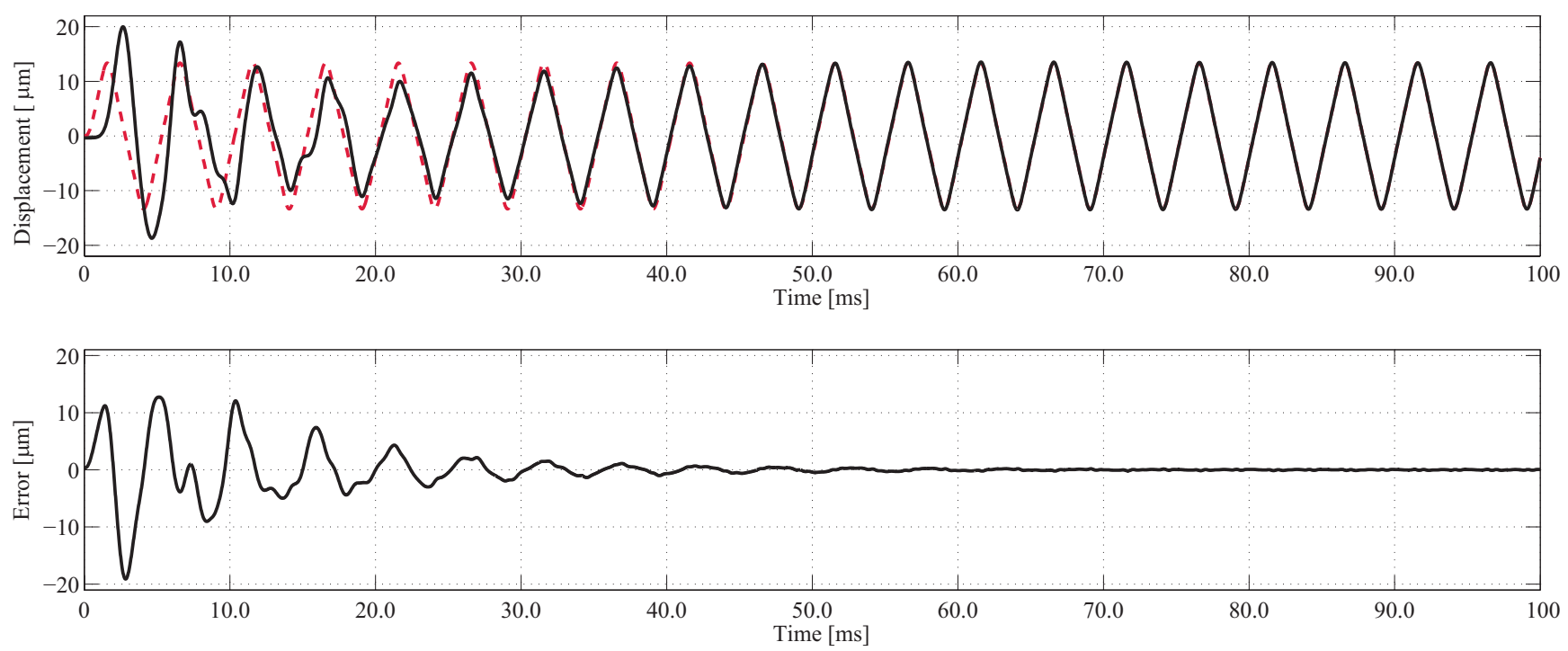

(a) Initial transient measured deflection $y_{m}$ and measured error $\varepsilon$.

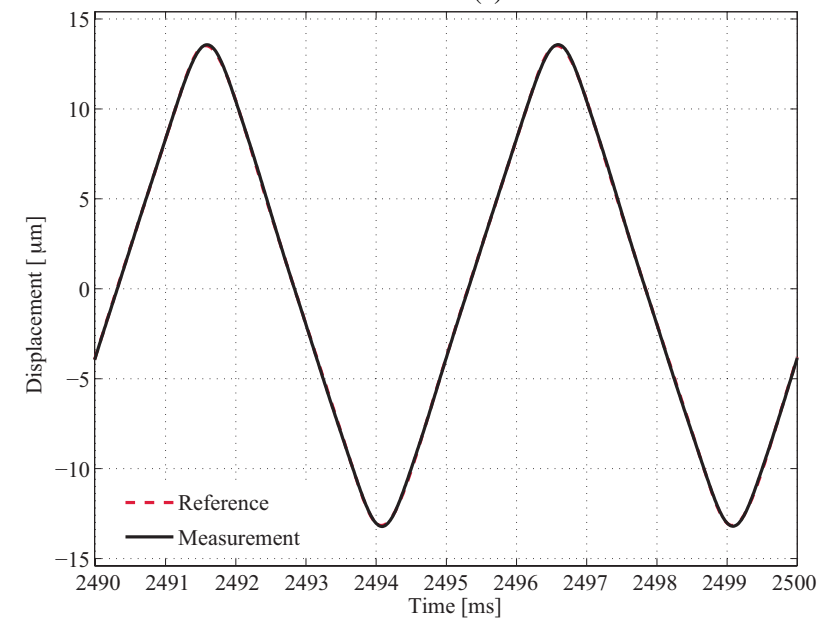

(b) Steady-state measured deflection $y_{m}$.

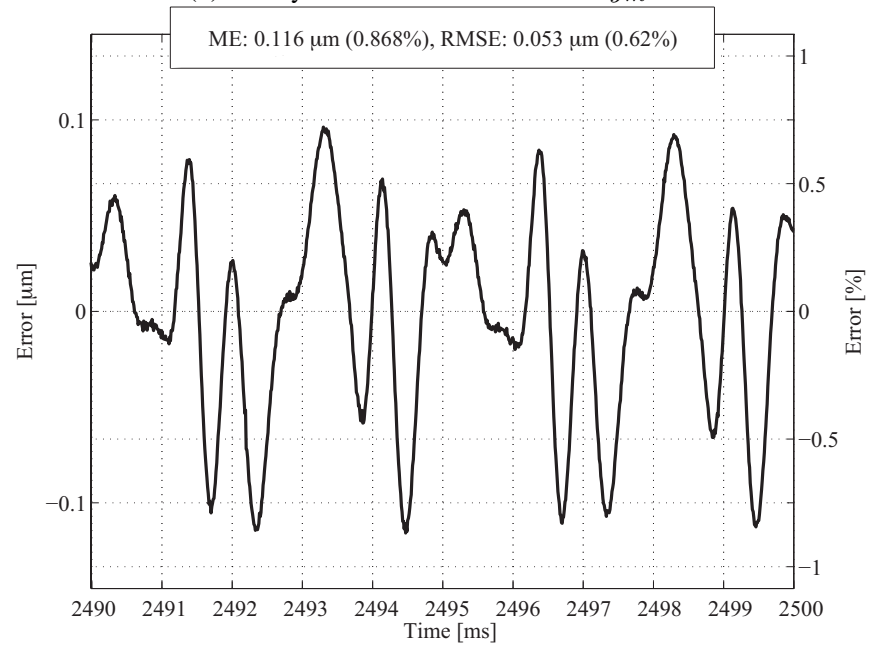

(d) Steady-state measured error $\varepsilon$.

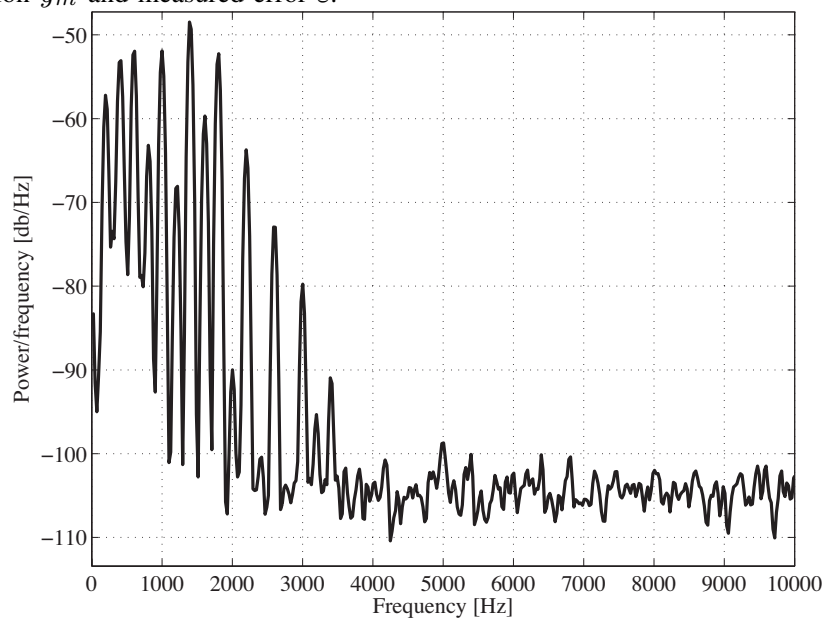

(c) Power spectral density estimate of error in steady-state.

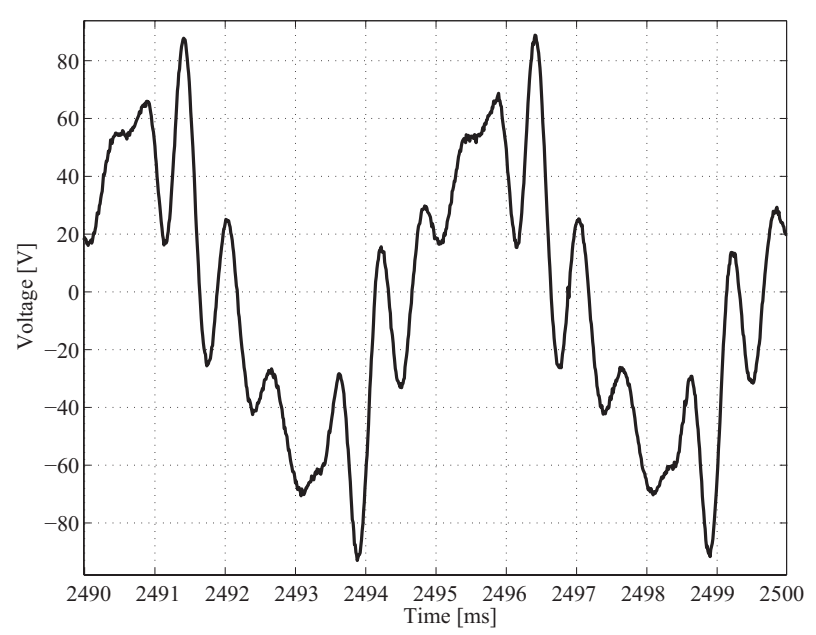

(e) Steady-state input voltage $u$.

Fig. 13: Reference at $200 \mathrm{~Hz}$ with 14.25- $\mu \mathrm{m}$ amplitude. 